\title{
Trends on Integrating Technologies for Mobile and Personalized Care
}

\section{Findings From the Section on Health and Clinical Management}

\author{
N. Bricon-Souf', M.-C. Jaulent ${ }^{2}$, Section Editors for the IMIA Yearbook Section on Health and \\ Clinical Management \\ 1 University of Toulouse - IRIT, Castres France \\ ${ }^{2}$ INSERM, UMR S872, EQ20, Paris, France
}

\begin{abstract}
Summary
Objectives: Summarize current excellent research and trends in the field of Health and Clinical Management.

Method: Synopsis of the articles selected for the IMIA Yearbook 2013.

Results: Five papers from international peer-reviewed journals have been selected for the section of Health and Clinical Management.

Conclusions: The selected articles illustrate current research regarding the Health and Clinical Management and showed that it is impacted by the importance of personalizing the care and integrating the use of mobile and connected technologies.
\end{abstract}

\section{Keywords}

Health and clinical management; patient care management; medical record systems; technologies in healthcare; telemedicine

Yearb Med Inform 2013:64-6

\section{Introduction}

Health and Clinical Management attempts to improve the efficiency of health care providers. The main challenges are, as reported in YB 2008[1], to improve quality, safety and efficiency of health and healthcare processes. Nevertheless, Information and Communication Technologies are still moving rapidly and are more and more integrated in nowadays practices. Health and clinical management systems need to introduce ICT tools in an efficient way onto the clinical practises. Two key aspects can be highlighted from the recent literature. One is the importance of the personalization and the contextualisation of the care processes, for example at home or to propose cross boundary health services. The other deals with the new opportunities offered by the mobile and connected technologies such as mobile phones.

\section{About the Paper Selection}

A comprehensive review of published articles in 2012 addressing the problem of the section Health and Clinical Management was performed. Table 1 lists the five papers selected as best papers for this section in the IMIA Yearbook 2013. The first paper [2] presents a deep reflexion on how to integrate different researches on Medical Informatics to model problem-oriented Clinical Notes. It deals with interoperability using HL7 standards and presents an experience conducted in daily practice. The second paper [3] focuses on the important topic of caring chronic diseases at home and at hospital, it reveals an interesting approach for a classification of the patient in order to personalize the monitoring according to the patient's condition. Third and fourth papers refer to the use of mobile phones for health care. The third paper [4] is a literature review enhancing different intervention strategies that occur during the use of mobile-phones for healthcare, and the potential impact to future researches. The fourth one [5] explores the potential of using SMS text messages to get free patient feedback about medication. It performs a deep description of a natural language processing process in order to extract and tag the medication information from the messages. The fifth article [6] investigates the different work practices in different countries and aims at using the obtained results in the design framework of a cross-boundary decision support system.

A brief content summary of these selected papers can be found in the appendix of this synopsis.

\section{Conclusions and Outlook}

The emergence of new technologies and the wide use of mobile technologies deeply impact the Health and Clinical Management. Innovations in health information technologies introduce different innovation perspectives for health care [7]. The best papers selected for the Health and Clinical Management section reflect the necessity of distributed and cooperative health care systems, offering the ability to take into account the actual practice 
and the use of mobile phones for health care interaction. Other challenges are emerging from our comprehensive review of current literature. The use of web 2.0 technologies becomes more and more integrated to clinical management and induces new health care management behaviours. We can firstly highlight the important trend of using mobile technologies and the issues linked to the use and the technologies of m-health systems [8], an interesting emerging aspect is the use of cloud computing in the health care area [9]. Finally, security challenges are addressed, in particular an important issue for this decade would be a better knowledge of the factors for ICT harm, as ICT problems arise as the number of medical ICT applications grow [10].

\section{Acknowledgement}

We would like to acknowledge the support of Martina Hutter and of the reviewers in the selection process of the IMIA Yearbook.

\section{References}

1. Kubias D. Health and clinical management--from hospital to home patient care management. Findings from the Section on Health and Clinical Management. Yearb Med Inform 2008:29-32.

2. Cillessen FH, de Vries Robbé PF. Modeling problem-oriented clinical notes. Methods Inf Med 2012 Dec 4;51(6):507-15.

3. Jeong S, Youn CH, Shim EB, Kim M, Cho YM, Peng L. An integrated healthcare system for personalized chronic disease care in home-hospital environments. IEEE Trans Inf Technol Biomed 2012 Jul;16(4):572-85

4. Klasnja P, Pratt W. Healthcare in the pocket: mapping the space of mobile-phone health interventions. J Biomed Inform 2012 Feb;45(1):184-98.

5. Stenner SP, Johnson KB, Denny JC. PASTE: patient-centered SMS text tagging in a medication management system. J Am Med Inform Assoc 2012 May-Jun;19(3):368-74.

6. Tawfik H, Anya O, Nagar AK. Understanding clinical work practices for cross-boundary decision support in e-health. IEEE Trans Inf Technol Biomed 2012 Jul;16(4):530-41.

7. Savage GT, van der Reis L. A Dutch and American commentary on IT in health care: roundtable discussions on IT and innovations in health care. Adv Health Care Manag 2012;12:61-74.

8. Silva LA, Costa C, Oliveira JL. A PACS archive architecture supported on cloud services. Int J Comput Assist Radiol Surg 2012 May;7(3):349-58.

9. Coiera E, Aarts J, Kulikowski C. The dangerous decade. J Am Med Inform Assoc 2012 JanFeb;19(1):2-5

10. Constantinescu L, Kim J, Feng DD. SparkMed: a framework for dynamic integration of multi-

Table 1 Best paper selection of articles for the IMIA Yearbook of Medical Informatics 2013 in the sections 'Health and Clinical Management'. The articles are listed in alphabetical order of the first author's surname.

\section{Section}

Health and Clinical Management

- Cillessen FH, de Vries Robbé PF. Modeling problem-oriented clinical notes. Methods Inf Med 2012 Dec 4;51(6):507-15.

- Jeong S, Youn CH, Shim EB, Kim M, Cho YM, Peng L. An integrated healthcare system for personalized chronic disease care in home-hospital environments. IEEE Trans Inf Technol Biomed 2012 Jul;16(4):572-85.

- Klasnja P, Pratt W. Healthcare in the pocket: mapping the space of mobile-phone health interventions. J Biomed Inform 2012 Feb; $45(1): 184-98$.

- Stenner SP, Johnson KB, Denny JC. PASTE: patient-centered SMS text tagging in a medication management system. J Am Med Inform Assoc 2012 May-Jun; 19(3):368-74.

- Tawfik H, Anya 0, Nagar AK. Understanding clinical work practices for cross-boundary decision support in e-health. IEEE Trans Inf Technol Biomed 2012 Jul; 16(4):530-41.

media medical data into distributed $\mathrm{m}$-Health systems. IEEE Trans Inf Technol Biomed 2012 Jan;16(1):40-52.

Correspondence to:

Nathalie Souf

IRIT-Elipse

ISIS- Campus universitaire

Rue Firmin Oulès

81104 Castres, France

E-mail: nathalie.souf@irit.fr

Appendix: Content Summaries of the Selected Best Papers for the IMIA Yearbook 2013, Section "Health and Clinical Management" ${ }^{\prime \prime}$

\section{Cillessen FH, de Vries Robbé PF \\ Modeling problem-oriented clinical notes Methods Inf Med 2012 Dec 4;51(6):507-15}

The objectives of the research is to integrate problem-oriented clinical notes inspired from the POMR (Problem-Oriented Medical Record) proposed by Weed in 1960 to an Electronic Health Record. HL7 version 3 is chosen as a standard for the modeling process. Diagnosis and matter of worry are taken into account to model progress notes. Three main concepts

\footnotetext{
The complete papers can be accessed in the Yearbook's full electronic version, provided that the article is freely accessible or that your institution has access to the respective journal.
}

are retained: Concern (information about the patient that needs attention), Condition (health condition such as disease, injury, health-related state) and Assessment (clinician's conclusions and working assumptions). A discussion group mainly composed with clinicians and health care professionals uses HL7 methodology specification in order to analyze and design the project. Simulations, prototypes and trials are performed in order to validate the model. The proposed model is patient-centered and generic, it is based on POMR and SOAP (Subjective, Objective, Assessment, Plan) and it refers to the HL7 V3 standard. Issues emerging from literature review as well as clinicians functional needs are taken into account: the relationships between the different progress notes components are especially studied and help the clinician's reasoning, different lists and views on concerns and conditions are proposed. A HL7-V3 progress note model integrating the SOAP concepts helps to document clinical reasoning. Acts are used for each assessment that refers to a condition, conditions are separated from concerns and working lists group acts for specific purposes. A clinical note application has been proposed from this model. It is integrated in daily practice by a lot of clinicians $(>1000)$ and other professionals $(>2500)$.

\section{Jeong S, Youn CH, Shim EB, Kim M, Cho YM, Peng L \\ An integrated healthcare system for personalized chronic disease care in home-hospital environments \\ IEEE Trans Inf Technol Biomed 2012 Jul;16(4):572-85}


Caring patient at-home or at-hospital with chronic diseases is a true challenge due to high rates of people suffering from it. It is particularly important to provide healthcare quality, especially in home-care environment. Health care systems have to be adapted to changing situations, the monitoring of the patient depends on the gravity of the disease and the equipment should be modified according to the patient condition. A system for personalized chronic disease care is proposed. A patient status classification method (PSCM) using medical examination and medical database classifies a patient into a five tiers system. Knowledge about the patient diseases as well as a disease database are used to identify complication and calculate the patient status. The PSCM method has been validated on patient data for patients suffering of diabetes. The integrated healthcare system for personalized chronic disease care proposes a 6 components healthcare server and is dedicated to four types of chronic disease services: mental stress factor service, e-re-number estimation-based heart disease detection service, metabolic disease identification service and ECG signal-analysis-based arrhythmia detection service. Different scenarii for these services are proposed. An evaluation has been performed in order to evaluate the impact of the system on the physician's workload and assesses the usability of such a system.

\section{Klasnja P, Pratt W}

Healthcare in the pocket: mapping the space of mobile-phone health interventions

\section{J Biomed Inform 2012 Feb;45(1):184-98}

Mobile phones are more and more used as health platform systems. Text messaging interventions, monitoring applications or patient's own tracking of activities are some of the nowadays health applications. This paper presents a taxonomy of the interventions implemented with mobile phones and presents health care perspectives in developing mobile phone technologies. A review of the literature is performed on papers dedicated to the use of mobile phone for health intervention. It helps to identified the following mobile phone technologies: SMS that are often used as reminders or to send customized feedbacks to a patient, cameras allowing to collect data with low effort, native applications such as telemonitoring of hypertension that often relay on specific API and hardware features of the phones, automated sensing implementing portable equipment (pedometers, electrocardiograms for instance) and internet access . Five intervention strategies are identified: tracking health information, involving the healthcare team, leveraging social influence, increasing the accessibility of health information, utilizing entertainment. Smart reporting and use of context, mobile phone games are some of the future research directions and will be helped by the improvement of phone technologies.

\section{Stenner SP, Johnson KB, Denny JC PASTE: patient-centered SMS text tagging in a medication management system}

\section{J Am Med Inform Assoc 2012 May- Jun; 19(3):368-74}

Millions of cellular phones are used all over the world and give a huge opportunity to propose patient-centered electronic medication systems. In particular the use of SMS text has been proven as efficient to improve medication management. In order to benefit from bidirectionnel text messaging, the patient's messages should be processed. The paper presents a Patient-centered Automated SMS Tagging Engine (PASTE). The MMH (My MediHealth) system proposes a medication scheduler, a medication administration record and a reminder engine using SMS text. The study focuses on how to improve this system to allow the patients to write spontaneously free SMS texts. A specific natural language processing process is proposed to deal with the particularities of SMS writing (such as abbreviation, use of phonetic marks, contextual ambiguity) and to find the medication concepts and the action concepts of the SMS text. Preprocessing and synonym replacement followed by syntactic and semantic tagging generates an XML output from the SMS text messages. In order to evaluate the accuracy of this system, 130 medication messages issued from a built corpus have been tagged by PASTE. 80 messages were used to train the system. The 50 messages used for testing the approach were compared to an expert gold standard review and gave promising results:
F-Mesure of $91,3 \%$ for medication names, $94,7 \%$ for medication terms and $90,4 \%$ for action terms. Extracting medication information from the patient medication messages seems a promising approach.

\section{Tawfik H, Anya 0, Nagar AK Understanding clinical work practices for cross-boundary decision support in e-health \\ IEEE Trans Inf Technol Biomed 2012 Jul;16(4):530-41}

New health systems and in particular integrated healthcare decision support systems should extend their work processes across workplace boundaries. The goal of the paper is to study clinical work practices across three areas: the United Kingdom, the United Arab Emirates and the Nigeria to propose effective design for integrated cross-boundary system and answer a dilemma between subsystems tailored toward local practices and shared purpose of care. A user-centered study was performed from the U.K., the U.A.E. and the Nigeria to identify the different factors that characterize clinical work practices. Significant differences in patterns of clinical practices and decision making were underlined by amount half of the participants of the questionnaire for each countries. The study allows to identify main factors for the perception of work practice boundaries such as domain location, socio-cultural factors, and identify the need to support situated practices, to co-construct meaning between the different workplaces to avoid lack of trust, the need to share guidelines and patient-centered care as goals remains the same across the different countries, the need for abstract description of work in order to match the abilities of the clinicians to decontextualize their work. The clinical practice concept is proposed for the conceptual design of the CaDHealth framework. The practice taxonomy distinguishes ontological practices with concepts of a task domain, stereotypical practices which inform about the routine actions of a workplace and situated practices which integrate circumstances information. Three main services are proposed by this framework: clinical practice modeling, context morphing and suggestion augmentation using web-based information. 